\title{
The traveling salesman problem: A hierarchical model
}

\author{
SCOTT M. GRAHAM \\ Purdue University, West Lafayette, Indiana \\ ANUPAM JOSHI \\ University of Maryland, Baltimore, Maryland \\ and \\ ZYGMUNT PIZLO \\ Purdue University, West Lafayette, Indiana
}

\begin{abstract}
Our review of prior literature on spatial information processing in perception, attention, and memory indicates that these cognitive functions involve similar mechanisms based on a hierarchical architecture. The present study extends the application of hierarchical models to the area of problem solving. First, we report results of an experiment in which human subjects were tested on a Euclidean traveling salesman problem (TSP) with 6 to 30 cities. The subject's solutions were either optimal or near-optimal in length and were produced in a time that was, on average, a linear function of the number of cities. Next, the performance of the subjects is compared with that of five representative artificial intelligence and operations research algorithms, that produce approximate solutions for Euclidean problems. None of these algorithms was found to be an adequate psychological model. Finally, we present a new algorithm for solving the TSP, which is based on a hierarchical pyramid architecture. The performance of this new algorithm is quite similar to the performance of the subjects.
\end{abstract}

Processing of spatial information is an important cognitive ability. It is involved in such cognitive functions as perception, memory, attention, navigation, and problem solving. The multitude and diversity of these functions have often led students of cognition to assume the operation of several different and independent mechanisms (modules) for spatial information processing, each mechanism subserving its corresponding cognitive function. However, this account, recently referred to by the picturesque name "bag of tricks" (Ramachandran, 1990), should not be accepted until more parsimonious explanations are rejected. In fact, the results of prior research seem to suggest that visual perception, memory, and attention do involve similar mechanisms. Specifically, all these mechanisms are based on a hierarchical architecture

This study was reported at annual meetings of the Society for Mathematical Psychology at Irvine, 1995, and Chapel Hill, 1996 (an abstract is published in the Journal of Mathematical Psychology, 40, 356, 1996), and at the Annual Interdisciplinary Conference in Jackson Hole, 1999. This work was partially supported by NSF Awards ASC 9404859 and CCR 9202536, AFOSR Award F49620-92-J-0069, and ARPA ARO Award DAAH04-94-G-0010. The authors thank Tim McNamara and two anonymous reviewers for useful comments and suggestions. A.J. is in the Department of Computer Science and Electrical Engineering at the University of Maryland. Correspondence concerning this article should be addressed to Z. Pizlo, Department of Psychological Sciences, Purdue University, West Lafayette, IN 47907-1364 (e-mail: pizlo@psych.purdue.edu). for representing and processing spatial relations. We will briefly review several studies illustrating this observation.

Following the pioneering work of Rosenfeld and Thurston (1971) on pyramid algorithms, a number of studies have shown that a hierarchical pyramid performing multiscale/multiresolution analyses is an adequate model for a number of visual processes, such as subitizing, size perception, mental size transformation, and figure -ground segregation. Van Oeffelen and Vos's (1982) CODE model was a two-layer pyramid that identified clusters of dots in an image. Each dot in the image was represented by a Gaussian function. The standard deviation of the function was a fraction of the distance to the nearest dot. When a threshold was applied to the sum of all the functions, one obtained contours representing clusters of dots. Van Oeffelen and Vos showed that these clusters are similar to groups of dots that subjects form perceptually in a subitizing task.

Pizlo, Rosenfeld, and Epelboim (1995) used a multiple layer pyramid to model mental size transformation and size perception. In this model, a stimulus has a number of representations on different levels of scale and resolution. The representations are obtained by blurring the original image with a Gaussian function. The layers that are closer to the top of the pyramid have fewer processing nodes, larger receptive fields, and lower spatial resolution. Depending on the task, the model chooses an adequate spatial resolution of the representation. In a size perception task, the model transforms the spatial resolu- 
tion (precision) of the representation from coarse to fine As a result, if more time is allowed, the model can estimate sizes with higher precision. This leads to the wellknown phenomenon of the speed-accuracy tradeoff. In a mental size transformation task, two figures having different sizes are to be compared with respect to shape. Before the comparison can be made, the representation of one of them has to be transformed, in order to match the relative resolutions of the representations. This leads to longer reaction times when the size ratio is greater.

A multiresolution pyramid is only a special case of a more general class of hierarchical pyramids. The representations on different layers of a pyramid can be produced by operations other than just blurring. An example of such a pyramid model has been described by Pizlo, Salach-Golyska, and Rosenfeld (1997). Their model was designed to detect contours in the image by selecting only those arrangements of dots that conformed to the constraints of proximity and smoothness. The model began by detecting parts of the contours, which were subsequently combined (connected) by using a hierarchical clustering process. The simulation results on detecting straight and curved lines in an image closely matched the psychophysical results.

Van Oeffelen and Vos's (1982) CODE model has been recently applied to visual attention (Logan, 1996). In this theory, objects that are present in the visual field are organized into clusters, whose parameters depend on the spatial distribution of the objects. The clusters are determined by a method described by van Oeffelen and Vos. Unlike their model, however, Logan's theory allows changing the sizes of the clusters (by changing the value of the threshold). The probability that attention will be directed to a given region (cluster) is determined by the height of the corresponding distribution above the threshold.

Another line of research implying hierarchical models for spatial information processing is related to spatial memory (e.g., McNamara, Hardy, \& Hirtle, 1989). By using several dependent variables (order of recall, time of recall, and errors in recalling distances), McNamara et al. showed that spatial memory is organized hierarchically. The higher levels in this model contain representations of larger clusters.

Clearly, this prior research on visual perception, attention, and memory implies a common underlying principle, which takes the form of a hierarchical structure with clustering operations. The claim that human spatial information processing may involve hierarchical structure is not completely surprising in view of the well-established fact that a pyramid algorithm tends to be an optimal architecture in various computational tasks (see Jolion \& Rosenfeld, 1994, for a recent review of pyramids). It remains to be verified whether hierarchical processing is also involved in other aspects of spatial information processing, such as solving spatial problems

In this paper, we test and model the mental mechanisms involved in solving a well-known spatial problem called the traveling salesman problem (TSP). The TSP is the task encountered when a salesperson, starting in a city, wishes to visit each of $(N-1)$ other cities exactly once and return to the starting city. The goal is to determine in what order the salesperson should visit the cities to minimize the total distance traveled. When the cities are points on a Euclidean plane, the problem is called the Euclidean TSP (E-TSP).

In the next section, we provide a review of prior research on the E-TSP in both engineering and psychology. Then, we present our experiment, followed by testing of prior algorithms. Finally, we present our new hierarchical pyramid model and describe the results of its testing.

\section{Prior Research on the Traveling Salesman Problem}

\section{Artificial Intelligence and Operations Research}

TSP belongs to the class of problems called NP complete. For this class, it is considered very unlikely that there exists an algorithm that will guarantee finding the optimal solution in time that is, at most, a polynomial function of the problem size (problem size refers here to the number of cities). This means that an algorithm guaranteed to produce optimal solutions for TSP would have to perform, in the worst case, an exhaustive search through all possible tours. An exhaustive search results in a number of operations that is an exponential function of the number of cities, which leads to extremely long solution times (see Lawler, Lenstra, Rinnooy Kan, \& Shmoys, 1992, for an overview of TSP research).

Because of the difficulty of determining the optimal solution, even for problems of moderate sizes, artificial intelligence and operations research ( $\mathrm{AI} / \mathrm{OR})$ researchers have investigated algorithms that produce approximate solutions. These approximating algorithms produce a solution quickly that is, in most cases, "good enough." More exactly, approximating algorithms produce solutions with a bounded error in time that is, at worst, a polynomial function of the problem size. A bounded error means that the worst solution produced by the algorithm has a length that will not exceed a known multiple of the optimal solution length. The lowest error bound found to date for a TSP-approximating algorithm is 1.5 times the optimal length (Christofides, 1976). The number of operations performed by this algorithm is a cubic function of the problem's size [in technical language, the computational complexity of this algorithm is $O\left(N^{3}\right)$ ].

Algorithms with a polynomial complexity have been shown to have a bounded error only for a TSP in which distances satisfy metric axioms (i.e., distances between different cities are greater than zero, are symmetric, and satisfy triangle inequality). Despite this limitation, approximating algorithms find many practical applications, perhaps because problems encountered in our everyday environment can often be adequately modeled by a metric (Euclidean) space. Below, we briefly describe several representative $\mathrm{AI} / \mathrm{OR}$ algorithms that produce approxi- 
mate solutions to E-TSP in a polynomial time. Later in this paper, the performance of these algorithms will be compared with the performance of human subjects.

A nearest neighbor $(\mathrm{NN})$ algorithm produces a tour by sequentially adding a connection from the most recent city visited to the nearest city that has not yet been visited. This procedure is repeated until all of the cities in the problem have been visited, at which time a final connection is made to the original/starting city. The algorithm uses each city in the problem as a start/end point to produce $N$ such tours and records the shortest solution. The computational complexity of this algorithm is $O\left(N^{3}\right)$ (Golden, Bodin, Doyle, \& Stewart, 1980).

A cheapest insertion $(\mathrm{CI})$ algorithm begins by creating a closed loop subtour, which visits only a subset of the cities in the problem. This subtour initially visits only a starting city and its nearest neighbor. The $\mathrm{CI}$ algorithm then sequentially inserts into the subtour the unvisited city that produces the least increase in the subtour length. The insertion procedure is repeated until all the cities in the problem are inserted. Again, all $N$ cities are used as the starting location, and the best solution is recorded. The computational complexity of the $\mathrm{CI}$ algorithm is $O\left(N^{3} \log N\right)$ (Golden et al., 1980).

A convex hull with cheapest insertion (CCI) algorithm begins with the boundary of the convex hull as a subtour (the convex hull is the smallest convex polygon that contains all the cities in the problem, either in its interior or on its boundary). The remaining cities are then inserted sequentially into the subtour, as in the $\mathrm{CI}$ algorithm. The heuristic used to identify the convex hull has a computational complexity of $O(N \log N)$. The overall complexity of CCI is $O\left(N^{2} \log N\right)$, because of the insertion procedure (Golden et al., 1980).

A convex hull with cheapest insertion and threesegment swap (CCI3) algorithm produces, first, a tour of all the cities, using the $\mathrm{CCl}$ algorithm. Then, as a postprocessing step, all three-segment reconfigurations of the tour are examined for a reduction in total tour length (Lin \& Kernighan, 1973). If a reduction in tour length is found, the process is repeated, starting with the shorter tour. When no further reductions in length are found in any of the three-segment reconfigurations, the algorithm stops. The computational complexity of the CCI3 algorithm, as reported by Golden et al. (1980), is $O\left(N^{3}\right)$.

An elastic net (EN) algorithm (Durbin \& Willshaw, 1987 ) begins by forming a circle of nodes about the center of mass of the cities. These nodes are free to migrate on the plane of the problem. However, they always remain sequentially ordered. Each node initially has a strong attraction to the two adjacent nodes and a weak attraction to the cities in the problem. These attraction forces are used in an iterative process to gradually change the position of each node. Over a period of many iterative steps, the attraction forces between the nodes and the cities increase, and the attraction forces between the adjacent nodes decrease. If the change in attraction forces and rate of positional change is small enough, the algo- rithm will cause the sequence of nodes to distort into a pattern that approximates a TSP solution. A tour of the cities can then be produced by identifying the city sequence that best matches the sequence of node locations. The computational complexity of the iterative process is $O\left(N^{2}\right)$. However, owing to the large number of iterations required, this algorithm has an extremely long execution time for larger problems.

It is worth pointing out that none of these algorithms involves hierarchical clustering, which was shown to be a critical component of human spatial processing. However, at this stage it is not clear whether this component is involved in human problem solving, either.

\section{Psychological Research}

There has been only a handful of studies that have provided results characterizing human performance in TSP, and none that have proposed a model of the underlying psychological mechanisms. In Michie, Fleming, and Oldfield's (1968) study, the subjects used an interactive display (interface) that recorded, stored, and retrieved one or more tours that the subject produced in the course of searching for a best solution. The results of 4 subjects and one algorithm (graph traverser) are reported on five Euclidean TSPs. Each of the five problems in their experiment consisted of 50 cities. The aided performance of the subjects (i.e., with the use of the interface) was found to be systematically better than the unaided performance. However, as Michie et al. pointed out, even the unaided performance was quite good, with 1 subject producing unaided solutions that were no more than $1.5 \%$ longer than the best, known solutions. This subject also produced aided solutions that were as short as, or shorter than, those produced by the graph traverser algorithm.

Consider now the study performed by MacGregor and Ormerod (1996). They tested the hypothesis that the difficulty of solving TSP is related to the number of nonboundary cities in a problem, rather than to the total number of cities. The term boundary city is used in their study to denote a city that is on the boundary of the convex hull. MacGregor and Ormerod's hypothesis was based on the following observations. First, the optimal solution to an E-TSP contains the same ordering of boundary cities as that found in the convex hull; any other ordering of boundary cities will create at least one tour segment crossing, and it is known that solutions that contain a tour crossing are nonoptimal. Second, the convex hull of an ETSP can be determined in a low-order polynomial time, $O(N \log N)$. Third, the human subjects almost never produce tour crossing. From this, MacGregor and Ormerod conjectured that humans begin solving TSP by finding the convex hull. As a result, the actual difficulty of a TSP would be related to the number of nonboundary cities in a problem, rather than to the total number of cities. MacGregor and Ormerod tested subjects on 10- and 20-city E-TSPs. They analyzed the subjective difficulty of the individual problems, using two different measures: counting the number of different solutions produced by the 
subjects and calculating a response uncertainty across the solutions. The response uncertainty, computed as $H=$ $\Sigma_{i} p_{i}\left(-\log _{2} p_{i}\right)$, represents the degree of randomness in the set of solutions observed (where $p_{i}$ is the probability of a given connection's being used). If all the subjects produce the same tour for a given problem, $H$ is equal to zero, and the problem is considered easy. On the other hand, if a given problem results in a random set of solutions, where each intercity connection occurs with equal probability, $H$ is maximal, and the problem is considered difficult.

MacGregor and Ormerod (1996) found that the subjects produced very good solutions: The mean solution lengths were less than $4 \%$ over optimal on the 10-city TSP and less than $10 \%$ over optimal on the 20-city TSP. Furthermore, for each of the 10-city TSPs, the optimal solution was produced by at least 1 subject. In their analysis of problem difficulty (randomness of solution), MacGregor and Ormerod found a significant correlation between the number of different solutions produced by the subjects and the number of nonboundary cities in the 10-city problems. This correlation, however, was not significant in the case of 20-city problems (MacGregor and Ormerod conjectured that, in larger problems, subjective difficulty depends more on the proximity among interior cities). The response uncertainty, on the other hand, was found to correlate significantly with the number of nonboundary cities for both the 10- and the 20-city problems.

In a recent study, Ormerod and Chronicle (1999) extended the results of MacGregor and Ormerod (1996). Their main conclusion, derived from the results of four experiments, was that human subjects begin the process of formulating a solution by global analysis, which may involve Gestalt rules of perceptual organization (good continuation, closure).

To summarize, the results presented in the studies by Michie et al. (1968), MacGregor and Ormerod (1996), and Ormerod and Chronicle (1999) show that human subjects are quite good at producing near-optimal solutions to the E-TSP. They furthermore indicate that the convex hull may be involved in the processes underlying human problem solving. These studies, however, do not provide enough information to formulate a computational model of the human mental processes involved in solving the TSP. The experiment presented in this paper was designed to provide such information. We tested both experienced and naive subjects on the E-TSP with $6,8,10,20$, and 30 cities, and we compared the performance of the subjects with the performance of AI/OR-approximating algorithms. Since two of those algorithms use the convex hull as the first stage in solving the TSP, the comparison of the performance of these algorithms with that of human subjects therefore provided a test for the psychological plausibility of the convex hull. None of the algorithms tested was found to be a psychologically possible model. We therefore formulated a new algorithm, which is based on a hierarchical architecture. As was pointed out in the introduction, this architecture has already provided adequate explanations for a range of cognitive functions related to spatial processing. We will show that a hierarchical architecture also leads to a plausible model of the mechanisms involved in solving the E-TSP.

\section{Testing Human Subjects on the Euclidean Traveling Salesman Problem ${ }^{1}$}

\section{Method}

Subjects. The three authors served as subjects in all of the conditions in this experiment. In addition, 3 other subjects, who were naive with respect to our hypotheses, were tested in most of the conditions. The naive subjects were graduate psychology students not involved in visual or problem-solving research.

Stimuli. TSP problems with $6,8,10,20$, and 30 cities were displayed on a computer monitor. The stimuli were viewed with both eyes from a normal viewing distance. The cities were represented on the screen by small circles, and the connections between the cities were represented by straight-line segments. The locations of the cities in each problem were generated randomly from a uniform distribution. The subjects were informed that the distances between the cities were equal to the Euclidean distances displayed on the monitor. A subject identified his or her solution (tour of the cities) by sequentially clicking a mouse pointer on the cities. As cities were selected, their color changed from blue to red, and the existing path, from the starting city to the current city, was indicated as a polygonal line. A tour was complete when all the cities had been visited and the first city was again selected. The subjects were free to start their solutions at any city in the problem. The subjects could change their solutions by undoing the last segment in a current solution (recursively) or by clearing the entire solution and starting over. The tour produced by the subject and the time used to produce the tour were recorded.

Procedure. The experiment was conducted in two stages. The first stage involved small problems $(6,8$, or 10 cities $)$, and the second stage involved larger problems $(10,20$, or 30 cities $)$. In the first stage, each of the authors was tested in nine sessions, each session consisting of 20 problems of a given size (the same problems were used for all the subjects). The naive subjects were tested in only three sessions. The order of sessions was random and different for each subject. Each of the authors was instructed to use a varying amount of time in producing their solutions. Three time deadlines were used $(2,3$, and 4 sec per city). At the end of the time allocated for each trial, a tone was presented to the subject. This tone, however, did not terminate a trial. The deadline procedure was included as a factor in this experiment to test whether longer time would result in better solutions (shorter tours). Since we failed to find any systematic effect of the time deadline on the length of the solutions produced by the authors, the 3 naive subjects were tested without the use of the deadline. This allowed a reduction in the number of sessions in the case of the naive subjects. The naive subjects were instructed to use as much time as needed in order to produce a best solution. They were provided with one practice session containing examples of all the problem sizes. The authors, on the other hand, received extensive practice before the experiment.

In the second stage of the experiment, each subject was tested in three sessions, each session consisting of 30 problems of a given size. All the subjects were tested on the same set of problems, with the order of sessions different for each subject. All the subjects were instructed to use as much time as needed to produce a best solution. 


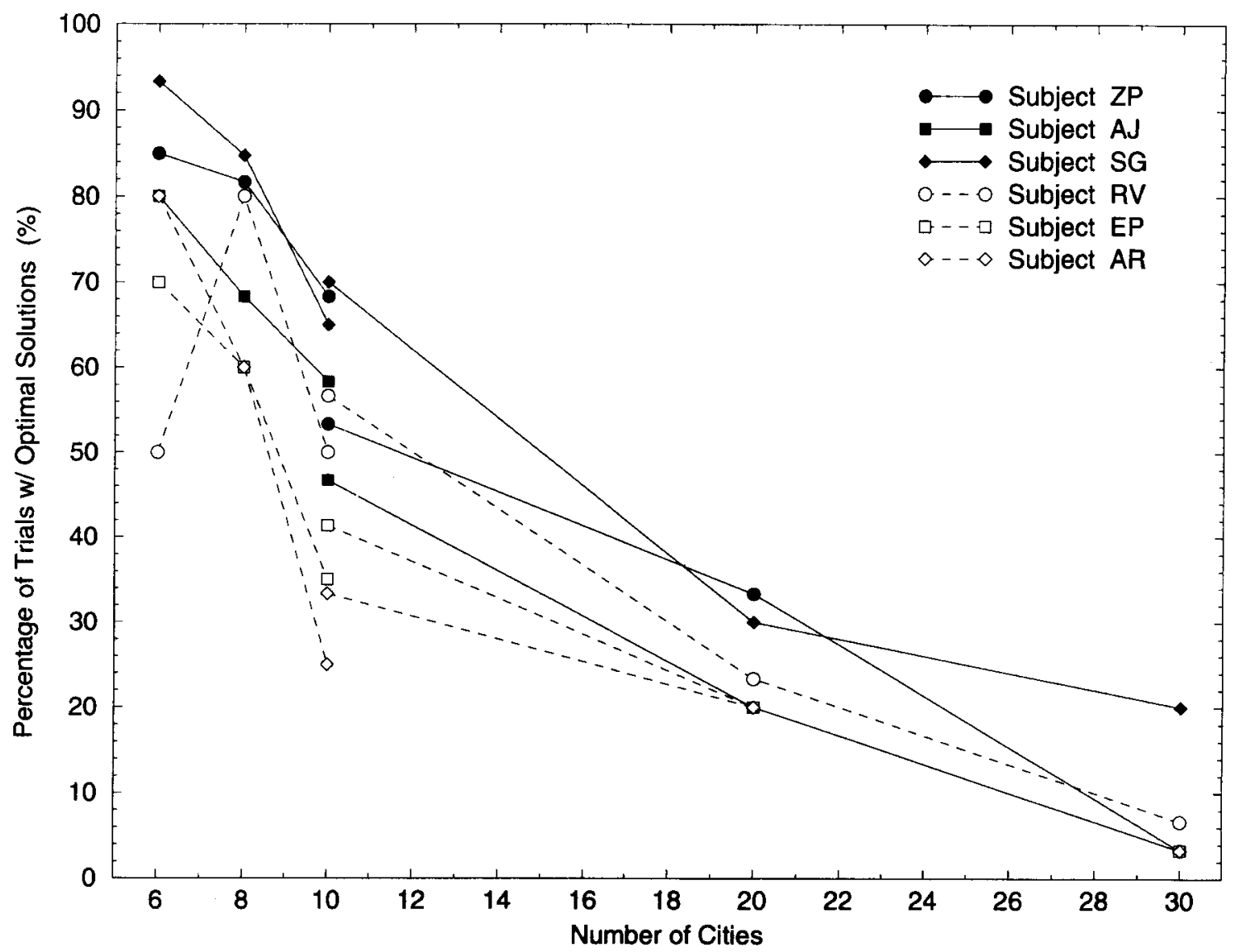

Figure 1. Percentage of trials on which the tour produced by the subject was optimal, as a function of the problem size.

Analysis. The solutions produced by the subjects were evaluated by taking the ratio of the length of the tour produced by the subject to the length of the optimal (shortest) tour. This ratio is referred to in this paper as the normalized tour length. The term solution error refers to the difference between the normalized tour length and 1.00. Optimal solutions were determined by a branch-and-bound algorithm. ${ }^{2}$

\section{Results and Discussion}

The performance of the authors was, on average, similar to but slightly better than that of the naive subjects. Furthermore, the authors were more consistent as subjects, and thus, their data show less variability. The better performance of the authors can be attributed to greater familiarity with the task.

A tour crossing was found in only one solution out of the 1,260 tours produced by the 6 subjects. Recall that MacGregor and Ormerod (1996) also found a small proportion of tour crossings. The absence of tour crossing indicated, according to MacGregor and Ormerod, that subjects use the convex hull in the process of solving TSP.

Figure 1 shows the percentage of optimal solutions produced by the subjects, as a function of the problem size. It is seen that in the case of small problems, the subjects produced an optimal solution in a large proportion of the problems. As the size of the problems increased, the subjects' ability to produce optimal solutions deteriorated gradually. Figure 2 shows the effect of the problem size on the normalized tour length. It can be seen that the average normalized tour length is very close to optimal and is not strongly affected by problem size. The differences in performance among the subjects are comparable with the standard errors. The variability of performance of the naive subjects is greater in the case of small problem sizes. This is likely to be related to the limited familiarity with the task, since the subjects started with these problems. This lack of familiarity is probably also responsible for the fact that the naive subjects tended to produce worse solutions (larger errors) in their first session, regardless of problem size (R.V. started with a 6-city, E.P. with an 8-city, and A.R. with a 10-city problem).

Figure 3 shows the effect of the problem size on the time per city used in producing the TSP solutions. It is seen that the time per city did not systematically depend on the number of cities in the problem. A constant time per city means that the total time used in solving the ETSP is, on average, a linear function of the number of cities. This linear relationship suggests that the underlying mental processes have a rather low complexity. This, in 


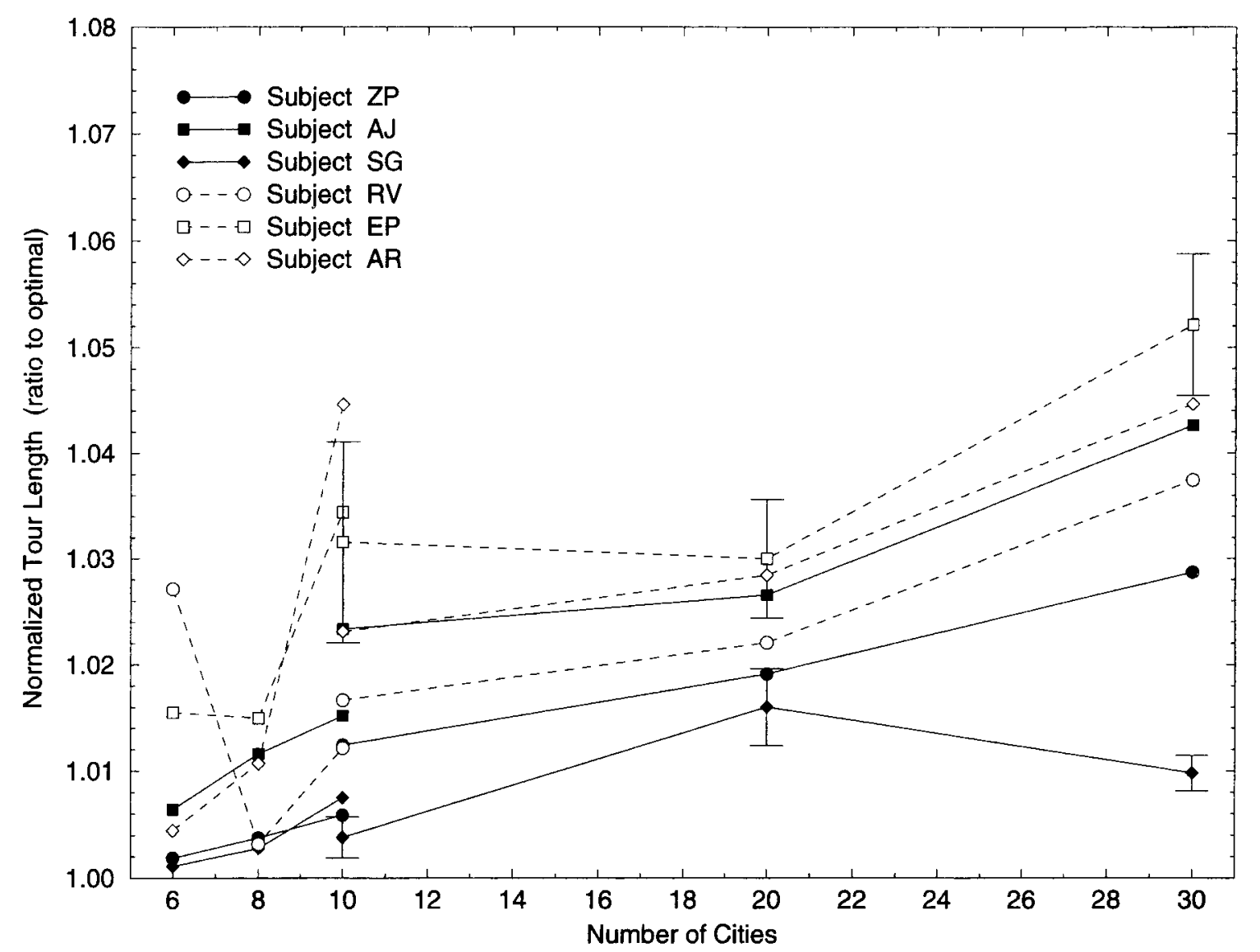

Figure 2. The normalized tour length as a function of problem size. Error bars ( \pm 1 standard error) are shown for the subjects with the best and the worst performances, for problem sizes 10,20 , and 30 . Variability in the case of smaller problems was similar.

turn, suggests that the subjects were not searching through the entire, or even a substantial portion of, TSP problem space. In the case of the authors, the time per city was shorter for smaller problem sizes than for larger ones. This result is simply related to the fact that in the first stage of the experiment (small problem sizes), the authors were tested with the time deadline procedure.

To summarize, the results of this experiment demonstrate that human subjects are quite adept at producing good solutions to the E-TSP. Furthermore, subjects appear to use procedures that have a processing time that is a linear function of the problem size [computational complexity $O(N)$ ]. This performance will next be compared with that of five representative AI/OR algorithms. The comparison seeks to determine whether any of the algorithms is a possible model of human mental processing.

\section{Comparing Performance of Artificial Intelligence/Operations Research Algorithms With That of Human Subjects}

The five AI/OR algorithms, which were described in the Artificial Intelligence and Operations Research sec- tion, were tested using the problems from the psychological experiment presented in the Testing Human Subjects on the Euclidean Traveling Salesman Problem section. These five algorithms use methods that range from very simplistic $(\mathrm{NN})$, to more sophisticated (CCI3, EN). Therefore, at least some of the algorithms should produce performances comparable with that of human subjects. Two of these algorithms (CCI and CCI3) use the convex hull as the first stage of processing. Thus, this simulation experiment will also test the psychological plausibility of the convex hull (recall that the results of prior research led to the conclusion that human subjects find and use the convex hull in solving the E-TSP).

Figure 4 shows the percentage of optimal solutions produced by the algorithms, as a function of the number of cities in the problem. Also shown in this graph is the averaged percentage of optimal solutions for the authors and the naive subjects, as well as a $99 \%$ confidence interval for the mean performance of human subjects at each problem size. For problems with 6-10 cities, the number of optimal solutions produced by the $\mathrm{NN}$ and $\mathrm{CI}$ algorithms was comparable with that produced by the subjects. The remaining algorithms produced substantially more optimal solutions. 


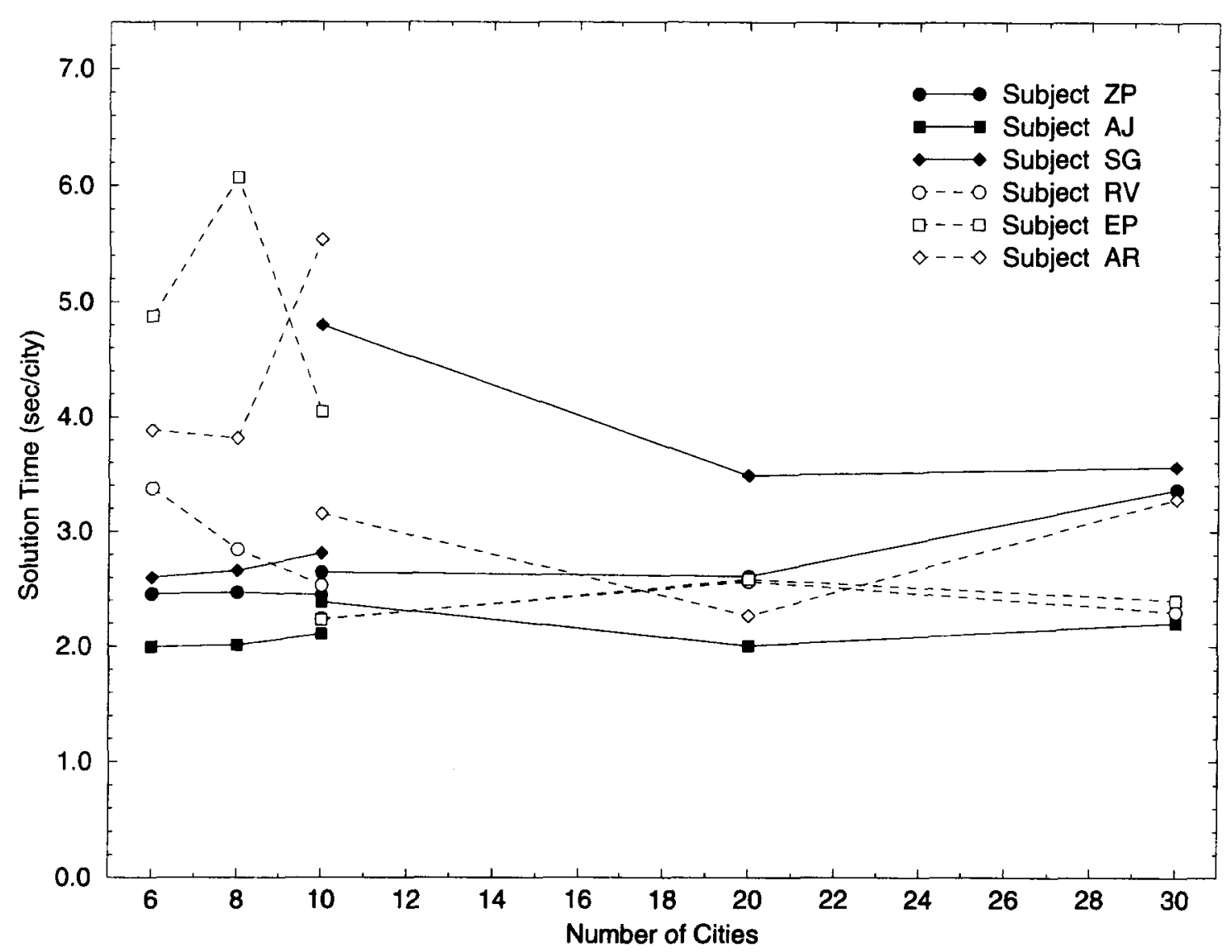

Figure 3. Solution time (seconds/city) as a function of problem size.

For larger problems ( 20 and 30 cities), only the CCI algorithm produced a number of optimal solutions comparable with that produced by the subjects. Overall, none of the five algorithms produced results within the confidence intervals for all the problem sizes, which suggests that none is an adequate psychological model.

Figure 5 shows the normalized tour length as a function of the number of cities in the problem (confidence intervals are also shown in this graph). Again, none of the algorithms has a performance that could be considered comparable with that of the subjects over the full range of problem sizes tested (for the CCI algorithm, the data points are within the confidence intervals, but in the case of problems sizes 6-20, the data points are at the lower extremes of the intervals).

On the basis of these results, as well as on the basis of the fact that all these algorithms have computational complexity greater than $O(N)$ [recall from the previous section that human subjects demonstrated the complexity $O(N)$ ], we conclude that none of the five algorithms tested is an adequate model of the mental processes involved in solving the E-TSP. Since two of these algorithms use the convex hull, we can further conclude that it is not likely that humans solve the TSP by finding and using the convex hull, as has been suggested by prior psycho- logical research. This conclusion is not completely surprising. Note that the convex hull has a quite limited applicability: It is a potentially useful feature only for the ETSP. In other spatial problems, such as finding a shortest path between a start and a goal, the convex hull is not likely to lead to an efficient algorithm. Hierarchical clustering is much more general. In fact, it has already been demonstrated that hierarchical clustering is a critical element in several cognitive functions, such as perception, memory, and attention (see the introduction). Interestingly, when we asked the subjects to describe the strategies that they used, they reported that it seemed natural to visit, one after the other, the cities that were spatially close to one another. According to the subjects, solving a problem involved first establishing groups (or clusters) of cities, then a tour of the clusters, and finally the sequence within each cluster. This description is perfectly consistent with the idea of hierarchical clustering. As was pointed out in the introduction, hierarchical clustering can be implemented very efficiently by pyramid algorithms (Jolion \& Rosenfeld, 1994; Pizlo et al., 1997; Scheessele, Graham, \& Pizlo, 1996). The following is a brief description of pyramids.

A pyramid consists of a number of layers, each layer containing nodes. These nodes are independent process- 


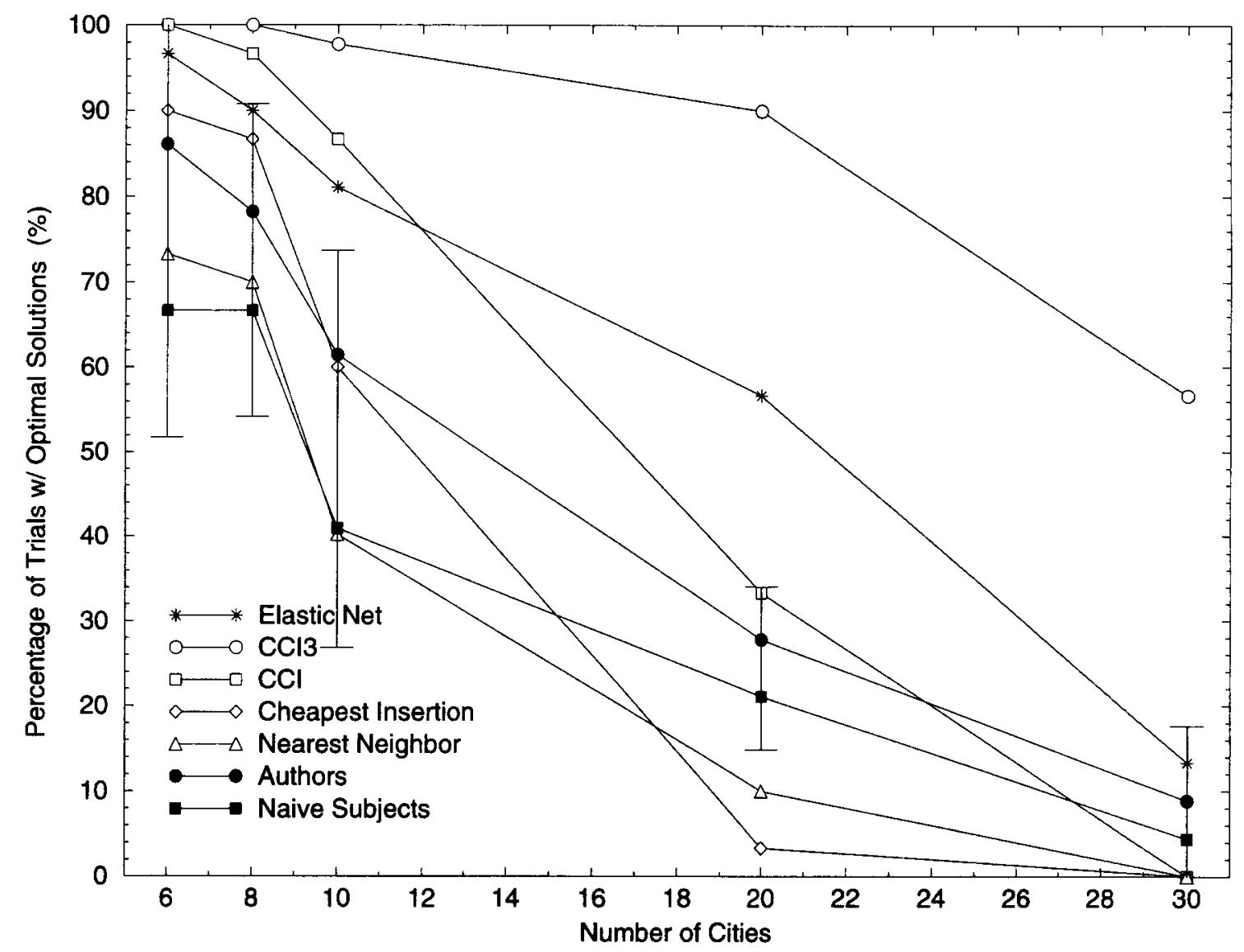

Figure 4. Percentage of trials on which the solution produced by artificial intelligence/operations research algorithms was optimal. These results were obtained using the problems on which the subjects were tested. The mean performance of the authors and the naive subjects is also shown. The results on the 10-city TSP have been averaged. The vertical bars represent $99 \%$ confidence intervals.

ing elements with links to parent nodes (next higher layer), neighbor nodes (same layer), and child nodes (next lower layer). Specifically, for a nonoverlapping quadpyramid, each node (or cell) is linked to one parent node, four neighbor nodes, and four child nodes. The bottom layer (layer 0 ) has $n$ nodes. The first layer above this has $n / b$ nodes. Layer $k$ has $n / b^{k}$ nodes. The parameter $b$ is called the reduction ratio ( $b=4$ for a nonoverlapping quad-pyramid). A pyramid has several distinctive characteristics: (1) It has a hierarchical organization, which preserves topographical mapping; (2) each node processes (locally) information received from a portion of the image (the node's receptive field); (3) receptive field size increases as one progresses upward in the hierarchy; (4) the pyramid can process information in a parallel fashion, with the nodes on a given level operating simultaneously and independently; and (5) it can process information in a bottom-up, as well as a top-down, direction. Interestingly, all of these properties closely resemble the known neuroanatomical and neurophysiological properties of the human visual system (Van Essen \& Anderson, 1995).
In the next section we describe an algorithm for solving the E-TSP that is based on a pyramid architecture. The performance of this algorithm is then compared with that of the subjects.

\section{A Pyramid Algorithm for Solving the Euclidean Traveling Salesman Problem}

\section{Description of the Algorithm}

A TSP problem is initially represented in the algorithm as a high-resolution image in the bottom layer of a pyramid. In this image, each city has the size of a single array cell and is represented by an intensity value of $2^{20} / N$. All the other cells in the array receive an intensity value of zero (thus, an average intensity is 1.0 ). A hierarchical series of images is then produced by a bottom-up process, which blurs and compresses the original image. This blurring and compression are performed by convolving the image from the next lower layer with a two-dimensional Gaussian filter and expressing the result as an image with a reduced resolution (Jolion \& Rosenfeld, 1994). 


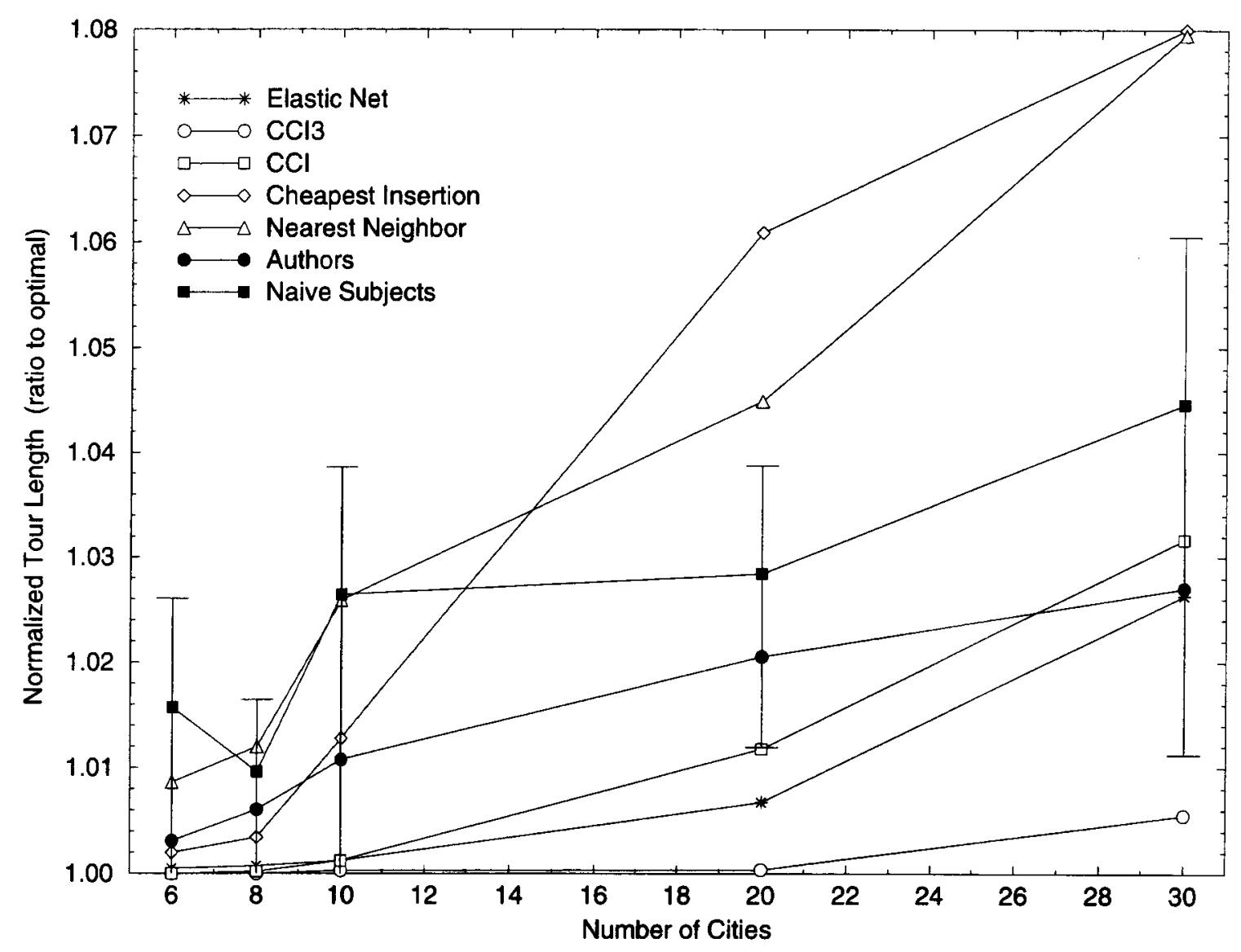

Figure 5. Normalized tour length produced by the artificial intelligence/operations research algorithms as a function of the problem size. These results were obtained using the problems on which the subjects were tested. The mean performance of the authors and the naive subjects is also shown. The results on the 10-city TSP have been averaged. The vertical bars represent $99 \%$ confidence intervals.

This procedure results in a hierarchy of images, with the first image having $N$ sharp peaks and subsequent images representing intensity distributions with a decreasing number of modes.

The algorithm interleaves three quad-pyramids to simulate an architecture known as the fractional pyramid (Burt, 1981). A fractional pyramid was used because the reduction ratio of a standard quad-pyramid $(b=4)$ was found to be too large (specifically, the quad-pyramid occasionally produced solutions with large errors). By using three quad-pyramids, our algorithm approximates a fractional pyramid with a reduction ratio of $b=4^{1 / 3}$.

After producing a representation of the problem on each layer, the algorithm identifies the location of the modes in the intensity distributions. The modes in each layer of the pyramid are then treated as cities, forming a hierarchy of problems, which are simplified representations of the original TSP. Because the problems in this hierarchy are topographically similar, only a small difference in the solution is expected for representations on adjacent layers. A solution to the original problem can therefore be produced efficiently by starting with a tour of the modes on a layer high in the pyramid and sequentially improving this tour approximation in a top-down process. This process starts at the lowest layer in the pyramid at which there are no more than three modes. In other words, at this resolution, cities belong to, at most, three clusters. A unique tour of these modes is produced and used as a first approximation of the TSP solution. A tour of the modes in each progressively lower layer is then produced by inserting the modes found in that layer into the tour produced on the previously higher layer. Once all of the modes on the current layer are inserted, the modes from the previous layer are removed. This top-down sequence of tour improvement produces a TSP solution on the bottom layer of the pyramid (where the image representation matches that of the original problem). The insertion method used in this algorithm is a simplified version of that used in the CI algorithm. It consists of two steps. First, the mode locations on a given layer are ordered according to their intensity levels. Then, these modes are inserted into the tour in descending order of their intensity, so as to produce the minimum increase in tour length. The number of operations performed by this 
$0 \quad 100200300400500600$

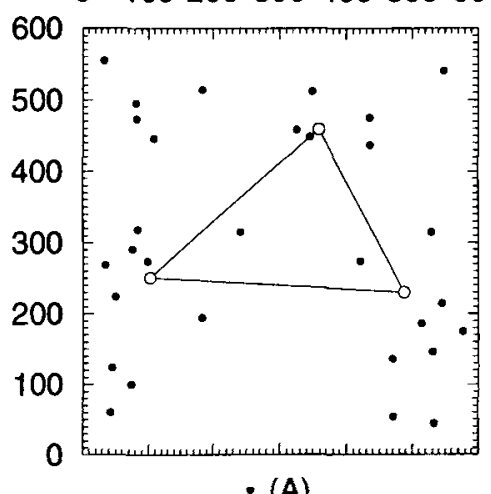

- (A)

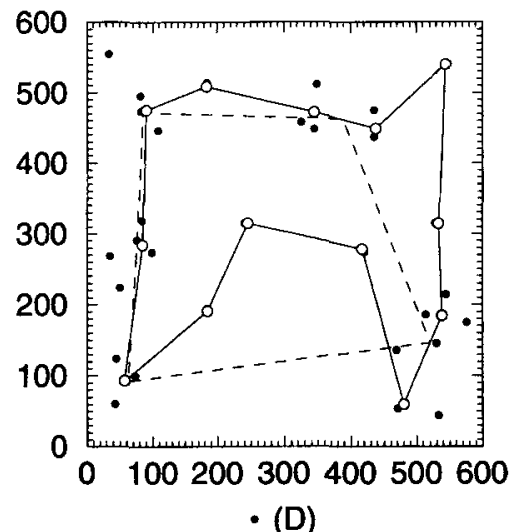

0100200300400500600

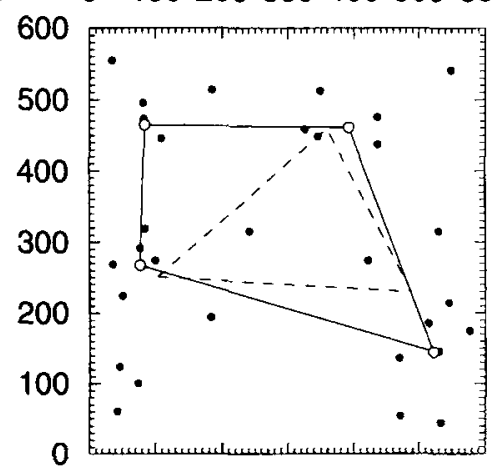

- (B)

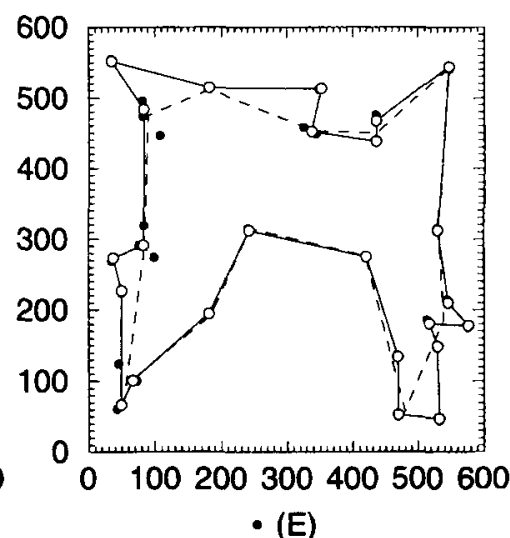

0100200300400500600
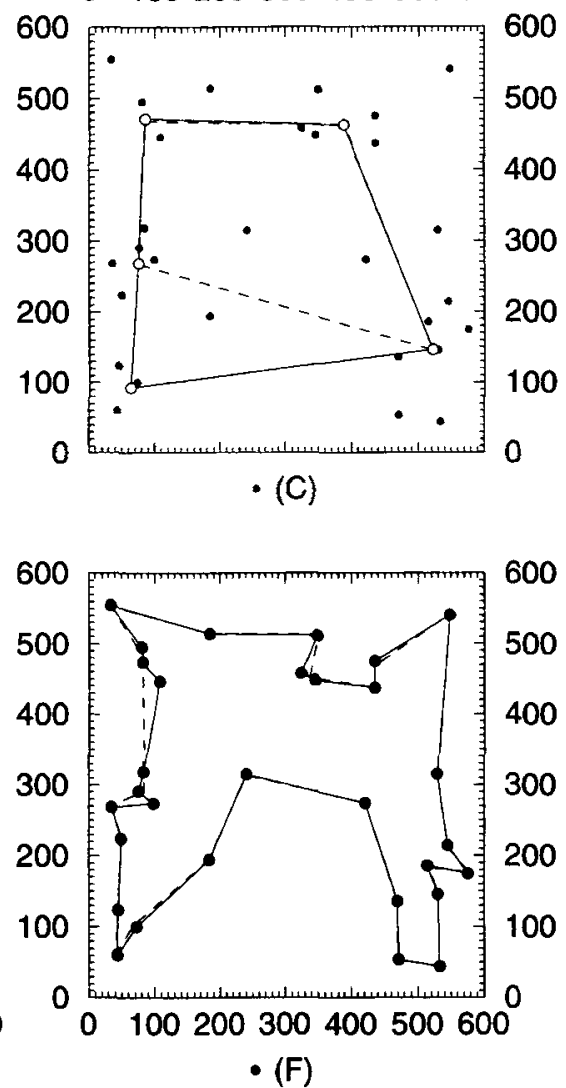

Figure 6. Illustration of the process of tour estimation performed by the pyramid algorithm. Filled circles show the original traveling salesman problem (TSP), open circles the location of modes in a particular layer of the pyramid, solid lines the current tour approximation, and dashed lines the prior tour approximation. Panel $A$ shows the tour of three modes on Layer 19 of the pyramid, panel $B$ the first major improvement in tour estimation, panels $C$, D, and E subsequent tour estimate improvements, and panel $F$ the final TSP tour.

algorithm in its current implementation is a quadratic function of the problem size (see the Appendix). Our discussion of the possible elaboration of this algorithm, presented in the Summary and Discussion section, indicates that the complexity of this algorithm can be reduced to $O(N)$, which is identical to the complexity of the mental mechanisms.

Figure 6 illustrates the top-town process of tour improvement for a 30 -city problem. The initial tour approximation is shown in Figure 6A. This tour has only a crude resemblance to a final solution of the problem. The tour improvement process involves two basic types of change: (1) the gradual migration of a mode location and (2) the decomposition of a mode from a higher layer into multiple modes in a lower layer. These two types of tour change can be easily seen in Figures $6 \mathrm{~B}$ and $6 \mathrm{C}$. The tour shown in Figure 6D has begun to take on a shape, which resembles a tour of the cities. This tour visits the 13 modes on Layer 15 in the pyramid. The tour shown in Figure 6E consists of 24 modes, and in Figure $6 \mathrm{~F}$ all the city locations have been incorporated into a final solution. (Please note that Figure 6 does not show all of the stages present in the tour improvement process. Several intermediate tour improvements have been omitted.)

\section{Testing the Pyramid Algorithm}

The pyramid algorithm was tested on the same problems as those that were used in the psychological experiment described in the Testing Human Subjects on the Euclidean Traveling Salesman Problem section. The algorithm produced only one tour crossing. This implies that the order of boundary cities in the algorithm's solutions is almost always the same as the order of the cities in the optimal solutions. This result is consistent with the results of human subjects reported in the section above, as well as in MacGregor and Ormerod (1996). The small proportion of tour crossings in subjects' solutions led MacGregor and Ormerod to the conjecture that subjects find and use the convex hull. Our simulations show that the absence of tour crossings in subjects' solutions can also be explained by hierarchical clustering.

Figures 7 and 8 show the performance of the pyramid algorithm. Also included in these figures is the averaged performance of the two sets of subjects, as well as the 


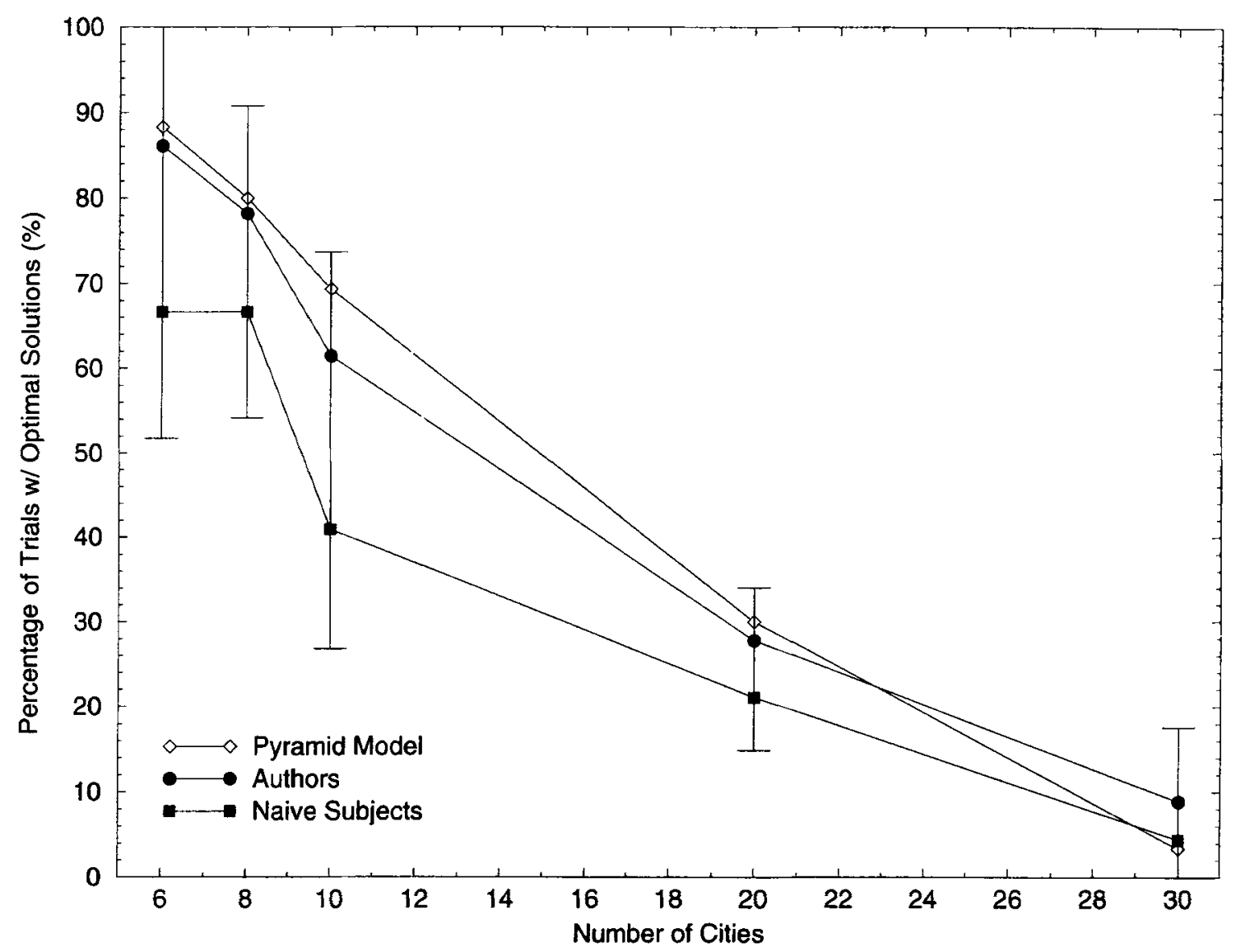

Figure 7. Percentage of trials in which the solution produced by the pyramid algorithm was optimal, as a function of the problem size. These results were obtained using the problems on which the subjects were tested. Also shown is the mean performance of the authors and the naive subjects. The vertical bars represent $99 \%$ confidence intervals.

confidence intervals. Both the normalized tour length performance and the proportion of optimal tours, for our algorithm, resemble quite closely those produced by the human subjects (the data points for this algorithm are within all confidence intervals).

We conclude that the pyramid algorithm is a possible (perhaps, even plausible) model of the mental processes involved in' solving the E-TSP. This conclusion is based on the following facts. (1) Performance of the algorithm (proportion of optimal solutions, solution error) is similar to that of the subjects. (2) The algorithm solves TSP by using grouping (clustering) operations similar to those described by the subjects. (3) The architecture and functioning of the algorithm are similar to known neuroanatomical and neurophysiological properties of the human visual system. (4) The computational complexity of the algorithm is of low order (i.e., not much greater than the linear time observed for the subjects).

We want to point out that some of the features of our algorithm are not entirely new; they have been explored to some extent in prior algorithms for the TSP. Below, we briefly discuss prior approaches that investigated the concepts of hierarchical clustering, local processing, and algorithms of low complexity.
Krolak, Felts, and Marble (1971) examined a manmachine synergy for producing solutions to large TSPs. The subjects in their experiment were provided with a computer-generated hierarchy, consisting of clusters of cities (and clusters of clusters), which were determined by an algorithm that solved the assignment problem. Their results showed that clustering according to the assignment problem was useful in assisting subjects to produce good solutions. Krolak et al., however, did not propose (or test) a model of how hierarchical clustering might be used by human subjects. Rather, their research focused on showing the "economic" feasibility of incorporating human subjects when solving large problems (as opposed to purely algorithmic solution that required costly computer resources).

The concept of clustering was used also by Karp (1977) in an algorithm that identified subgroups of cities according to a binary partitioning strategy. Karp's algorithm partitioned a problem (recursively) into regions at the median city; then it produced local tours for these regions, and finally it produced a global tour by combining the local solutions. Karp was primarily interested in investigating the relationship between solution time and worst case error for the algorithm. He did not test whether the algorithm is related to human mental processes. 


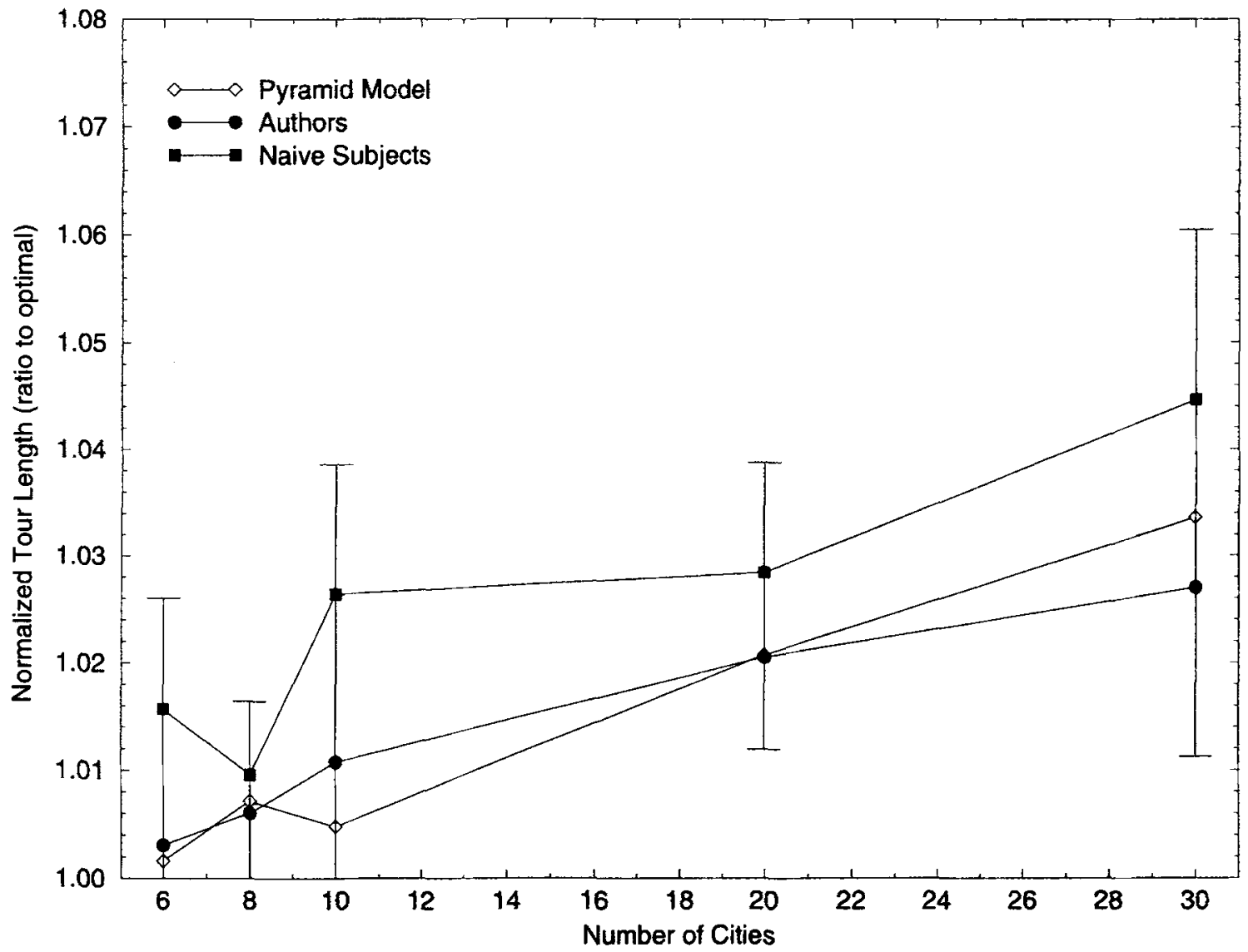

Figure 8. Normalized tour length for the pyramid algorithm as a function of problem size. These results were obtained using the problems on which the subjects were tested. Also shown is the mean performance of the authors and the naive subjects. The vertical bars represent $99 \%$ confidence intervals.

Finally, Platzman and Bartholdi (1989) examined the use of space-filling curves to produce TSP solutions in time that were a low-complexity function of the problem size $[O(N \log N)]$. A space-filling curve represents a transformation that is a one-to-one continuous mapping of a multidimensional space into a one-dimensional space. By translating the locations of the cities to be visited onto a space-filling curve, the task of identifying a tour becomes one of sorting in a single dimension. For a nonuniform planar TSP, Platzman and Bartholdi report that their algorithm produces an average error similar to that of the NN algorithm (approximately 15\% over optimal for a large TSP). Since we showed that the performance of human subjects is much better than that of the NN algorithm, it is unlikely that Platzman and Bartholdi's algorithm is a psychologically plausible model.

\section{Summary and Discussion}

The experimental results presented in the Testing Human Subjects section show that human subjects produce quite good solutions to the E-TSP. Furthermore, the subjects produce these solutions in time that is, on average, a linear function of the number of cities in the prob- lem [computational complexity $O(N)$ ]. In the Comparing Performance of $\mathrm{AI} / \mathrm{OR}$ Algorithms section, we examined the performance of five representative AI/OR algorithms and found that none is an adequate model of the mental processes involved in solving the E-TSP. A new algorithm was then presented in the A Pyramid Algorithm for Solving the Euclidean Traveling Salesman Problem section that uses a pyramid architecture and solves the E-TSP by using hierarchical clustering. This architecture is similar to the neuroanatomical and neurophysiological properties of the human visual system. Furthermore, this architecture was already shown to provide adequate explanations for a range of cognitive functions that involve spatial processing (see the introduction). The pyramid algorithm has a performance that is quite similar to that of the subjects. These results suggest that this new algorithm is a plausible model of the mental processes involved in solving the E-TSP. There are, however, several issues that remain open for future research.

First, we believe that the computational complexity of the pyramid algorithm can be reduced. Currently, the algorithm has a complexity of $O\left(N^{2}\right)$, which is caused by the serial implementation of the $\mathrm{CI}$ heuristic. However, 
this insertion process can be improved in two ways. First, the algorithm can be implemented on a system that performs parallel processing. This will reduce the complexity of the CI process to $O(N \log N)$. Second, because the pyramid architecture preserves topographical mapping, a further reduction in complexity is possible. Specifically, the CI heuristic can be restricted to a search within a neighborhood of the mode to be inserted. Note that the identification of modes within a given neighborhood can be performed in constant time, because a neighborhood can be established without performing global operations. Restricting the $\mathrm{CI}$ search to the neighborhood of a given mode will not greatly affect the average solution length of the algorithm, since the best insertion of a given mode is not likely to be in a remote portion of the tour. These two changes together will reduce the computational complexity of the algorithm to $O(N)$.

Next, consider the reduction ratio $b$ of the pyramid. Our informal experiments have shown that the pyramid algorithm is sensitive to the rate at which the resolution changes between layers. A large reduction ratio produces a small number of layers in the pyramid. This may lead to adjacent representations' being very different from one another (especially on layers where there are few modes). This in turn may lead to large errors in the final tour. A small reduction ratio, on the other hand, produces a large number of layers in the pyramid. Many of these layers would have representations that are almost identical (especially on layers with $N$ modes) and, thus, would contribute little to the solution process. This suggests that there may not be a single reduction ratio that is optimal for all problems. Instead, the optimal reduction ratio may depend on the given problem, and furthermore, it may have to change from layer to layer within the pyramid.

Finally, we think that the study of human performance should be extended to larger problem sizes. Using larger problem sizes will provide additional testing of (1) the low computational complexity of the mental processes involved in solving the E-TSP, (2) the adequacy of the pyramid model, and (3) the quality of human solutions (tour length) relative to $\mathrm{AI} / \mathrm{OR}$ algorithms. In this paper, we have shown that human subjects produce good solutions via a process that has very low complexity and that the mean solution error increases with problem size at a linear or less-than-linear rate. It is quite conceivable that at larger problem sizes, human performance will be better than that of the best AI/OR algorithm tested (CCI3). If this proves to be true, an algorithm that models human mental processes could be of benefit to artificial problemsolving systems.

\section{REFERENCES}

BURT, P. J. (1981). Fast filter transforms for image processing. Computer Graphics \& Image Processing, 16, 20-51.

ChRISTOFIDES, N. (1976). Worst-case analysis of a new heuristic for the traveling salesman problem (Tech. Rep. No. 388). Pittsburgh, PA: Carnegie-Mellon University, Graduate School of Industrial Administration.
DURbin, R., \& Willshaw, D. (1987). An analogue approach to the travelling salesman problem using an elastic net method. Nature, 326, 689-691.

Golden, B., Bodin, L., Doyle, T., \& Stewart, W. (1980). Approximate traveling salesman algorithms. Operations Research, 28, 694711.

JoLION, J. M., \& ROSENFELD, A. (1994), A pyramid framework for early vision. Boston: Kluwer.

KARP, R. M. (1977). Probabilistic analysis of partitioning algorithms for the traveling-salesman problem in the plane. Mathematics of $O p$ erations Research, 2, 209-224.

Krolak, P., Felts, W., \& Marble, G. (1971). A man-machine approach toward solving the traveling salesman problem. Communications of the $A C M, 14,327-334$.

Lawler, E. L., Lenstra, J. K., Rinnooy Kan, A. H. G., \& Shmoys, D. B. (1992). The traveling salesman problem. Chichester, U.K.: Wiley.

Lin, S., \& Kernighan, B. W. (1973). An effective heuristic algorithm for the traveling-salesman problem. Operations Research, 21, 498516.

LOGAN, G. D. (1996). The CODE theory of visual attention: An integration of space-based and object-based attention. Psychological Review, 103, 603-649.

MACGregor, J. N., \& Ormerod, T. (1996). Human performance on the traveling salesman problem. Perception \& Psychophysics, 58, 527 539.

McNamara, T. P., Hardy, J. K., \& Hirtle, S. C. (1989). Subjective hierarchies in spatial memory. Journal of Experimental Psychology: Learning, Memory, \& Cognition, 15, 211-227.

Michie, D., Fleming, J. G., \& Oldfield, J. V. (1968). A comparison of heuristic, interactive, and unaided methods of solving a shortestroute problem. Machine Intelligence, 2, 245-255.

Ormerod, T. C., \& Chronicle, E. P. (1999). Global perceptual processing in problem solving: The case of the traveling salesperson. Perception \& Psychophysics, 61, 1227-1238.

Pizlo, Z., Rosenfeld, A., \& Epel boim, J. (1995). An exponential pyramid model of the time-course of size processing. Vision Research, 35, 1089-1107.

Pizlo, Z., Salach-Golyska, M., \& Rosenfeld, A. (1997). Curve detection in a noisy image. Vision Research, 37, 1217-1241.

Platzman, L. K., \& Bartholdi, J. J. (1989). Spacefilling curves and the planar traveling salesman problem. Journal of the Association for Computing Machinery, 36, 719-737.

RAMACHANDRAN, V. S. (1990). Visual perception in people and machines. In A. Blake \& T. Troscianko (Eds.), Al and the eye (pp. 21 77). New York: Wiley.

Rosenfeld, A., \& Thurston, M. (1971). Edge and curve detection for visual scene analysis. IEEE Transactions on Computers, C-20, 562569.

Scheessele, M. R., Graham, S. M., \& Pizlo, Z. (1996). Exponential pyramid as a model of the human visual system. In E. J. Delp, J. P. Allebach, \& J. Kovacevic (Eds.), IEEE and IS\&T Proceedings of the Ninth Workshop in Image and Multidimensional Signal Processing (pp. 108-109). Los Alamitos, CA: IEEE Computer Society Press.

VAN ESSEN, D. C., \& ANDERSON, C. H. (1995). Information processing strategies and pathways in the primate visual system. In S. F. Zornetzer, J. L. Davis, \& C. Lau (Eds.), An introduction to neural and electronic networks (2nd ed., pp. 45-76). New York: Academic Press. van Oeffelen, M. P., \& VOS, P. G. (1982). Configurational effects on the enumeration of dots: Counting by groups. Memory \& Cognition, 10, 396-404.

\section{NOTES}

1. This experiment also contained nonmetric TSP problems. Performance on these problems was much poorer, as compared with that on E-TSP problems. These results are not reported here, since they are beyond the scope of this paper. They are presented in the master's thesis of the first author.

2. The branch-and-bound algorithm was kindly provided by R. J. Craig, Lucent Technologies. 


\section{APPENDIX}

The pyramid algorithm performs three main operations: problem initialization, production of the hierarchical representations, and production of the sequence of tour approximations. These three operations are analyzed next.

\section{Initialization of the Problem}

Clearing and allocating the pyramid memory. This is not a function of the size of the problem. Instead, it is a function of the maximum resolution in the pyramid-that is, the receptive field size at the bottom layer.

Clearing and allocating the Gaussian convolution masks. This is primarily a function of the selected Gaussian mask sizes.

Loading the problem into the pyramid. This is a process of $O(N)$ complexity, which is performed only once.

\section{Creating the Hierarchical Representations}

Blurring the image. This consists of the convolution of prior image representations with the appropriate Gaussian mask. This is not a function of the problem size but, instead, of the number of cells in the pyramid.

\section{Creating the Sequence of Tour Approximations}

Identifying the modes in the image representations. Here, each cell in the pyramid is examined, which results in a complexity that is a function of the pyramid size, but not of the problem size.

Ordering of the modes, by intensity, is a function of the size of the problem. Sorting can be done in $O(N \log N)$ time, and this bound can be improved if some a priori assumptions can be made about the data. This function is performed once per layer in the pyramid. The number of layers in the pyramid, however, is not a function of the size of the problem.

Creating an initial tour, which involves up to three modes. This is performed only once and is trivially done in constant time.

Creating the successive tours. Each improvement has a complexity of $O\left(N^{2}\right)$, since it involves a search for the best insertion of, at most, $N$ modes into a tour, with at most $2 N-1$ segments. This process is performed sequentially on each layer of the pyramid. The number of repetitions (i.e., the number of pyramid layers between the bottom layer and the layer with the initial tour estimate) is not a function of the problem size but, instead, is a function of the reduction ratio.

The computational complexity of the algorithm is $O\left(N^{2}\right)$. This complexity is primarily due to the tour approximation procedure. Note that this bound is not particularly tight and can be improved by using more efficient algorithms for the sorting and tour improvement phase. 